\title{
Identification, Cloning, and Expression of L-Amino Acid Oxidase from Marine Pseudoalteromonas sp. B3
}

\author{
Zhiliang Yu, Ning Zhou, Hua Qiao, and Juanping Qiu \\ College of Biological and Environmental Engineering, Zhejiang University of Technology, Hangzhou 310014, China \\ Correspondence should be addressed to Zhiliang Yu; zhiliangyu76@gmail.com
}

Received 25 August 2013; Accepted 22 October 2013; Published 9 January 2014

Academic Editors: P. Gyarmati, K. Hong, and F. Rodrigues

Copyright (C) 2014 Zhiliang Yu et al. This is an open access article distributed under the Creative Commons Attribution License, which permits unrestricted use, distribution, and reproduction in any medium, provided the original work is properly cited.

\begin{abstract}
L-amino acid oxidase (LAAO) is attracting more attentions due to its broad and important biological functions. Recently, an LAAOproducing marine microorganism (strain B3) was isolated from the intertidal zone of Dinghai sea area, China. Physiological, biochemical, and molecular identifications together with phylogenetic analysis congruously suggested that it belonged to the genus Pseudoalteromonas. Therefore, it was designated as Pseudoalteromonas sp. B3. Its capability of LAAO production was crossly confirmed by measuring the products of $\mathrm{H}_{2} \mathrm{O}_{2}$, a-keto acids, and $\mathrm{NH}_{4}{ }^{+}$in oxidization reaction. Two rounds of PCR were performed to gain the entire B3-LAAO gene sequence of 1608 bps in length encoding for 535 amino acid residues. This deduced amino acid sequence showed $60 \mathrm{kDa}$ of the calculated molecular mass, supporting the SDS-PAGE result. Like most of flavoproteins, B3-LAAO also contained two conserved typical motifs, GG-motif and $\beta \alpha \beta$-dinucleotide-binding domain motif. On the other hand, its unique substrate spectra and sequence information suggested that B3-LAAO was a novel LAAO. Our results revealed that it could be functionally expressed in E. coli BL21(DE3) using vectors, pET28b(+) and pET20b(+). However, compared with the native LAAO, the expression level of the recombinant one was relatively low, most probably due to the formation of inclusion bodies. Several solutions are currently being conducted in our lab to increase its expression level.
\end{abstract}

\section{Introduction}

L-amino acid oxidase (LAAO; EC 1.4.3.2) is usually dimeric flavoprotein containing a noncovalently bound FAD molecule as cofactor for each subunit. It is able to catalyze the stereospecific oxidative deamination of $\mathrm{L}$-amino acids to the corresponding a-keto acids with release of $\mathrm{NH}_{4}{ }^{+}$and $\mathrm{H}_{2} \mathrm{O}_{2}$ which is believed to be associated with its biological activities including inducing apoptosis, cytotoxicity, edema, hemolysis, hemorrhage, inducing or inhibiting platelet aggregation, and parasite-killing and antimicrobial activities [1]. When $\mathrm{H}_{2} \mathrm{O}_{2}$ is not degraded by catalase, it can cause a decarboxylation of the a-keto acid to the corresponding carboxylic acid.

This enzyme is widely distributed in different sources including snake venom [2], sea hare [3], insect drugs [4], algae [5], and microorganisms [6, 7]. So far, the LAAO from snake venom is the best characterized member of this enzyme family with respect to not only its toxicology but also its biochemistry, physiology, and medicine. In contrast, very little is known about LAAO from marine microorganism. Although various LAAO-coding sequences have been published to reveal that LAAO family members commonly have flavin as coenzyme and possess two conserved and characteristic sequence motifs, "GG" motif (RxGGRxxS/T) and dinucleotide-binding (DMB) motif ( $\beta$-strand $/ \alpha$-helix $/ \beta$ strand) [8], only some heterologous expression systems have been reported hitherto, probably due to the requirement of posttranslational modification of LAAO. In 2003, the first bacterial heterologous expression system for an LAAO from Rhodococcus opacus was reported. The lao-gene from $R$. opacus was cloned into Escherichia coli and Streptomyces lividans expression vectors. It was found that the expression in $E$. coli resulted in the accumulation of insoluble protein, but $S$. lividans was a suitable host for the heterologous expression of LAAO [9]. However, in 2008, the lao-gene from Streptococcus oligofermentans was successfully cloned and overexpressed in E. coli [10]. The LAAO isolated from sea hare was also functionally expressed in $E$. coli, but the expression level for soluble recombinant LAAO was relatively 
low, only ca. $0.2 \mathrm{mg} / \mathrm{L}$ culture medium [11]. To our knowledge, the expression of ophidian LAAO has rarely been achieved, except the successful expression of active recombinant LAAO in the methylotrophic yeast Pichia pastoris [12]. To date, the heterologous expression of LAAO is still a big challenge.

In this study, a yellow-pigmented LAAO-producing marine bacterial strain designated as Pseudoalteromonas sp. B3 on the basis of the physiological, biochemical, and molecular analysis was isolated. After retrieval of coding sequence, LAAO from Pseudoalteromonas sp. B3 (B3-LAAO) was cloned and functionally expressed in E. coli strain BL21 (DE3). This communication laid the foundation for further studies on enzymatic properties, structure, biological function, and application of B3-LAAO.

\section{Materials and Methods}

2.1. Sample Collection and Colony Isolation. The intertidal sediment samples were obtained from Dinghai sea area, Zhoushan, China $\left(30.03^{\circ} \mathrm{N}, 122.11^{\circ} \mathrm{E}\right)$. Each sample was collected at 50 to $100 \mathrm{~cm}$ depth below the sea surface. The samples were placed in special presterilized plastic bottles and brought to the lab in aseptic condition. After serial dilution (up to $10^{-6}$ dilution) using sterilized sea water, $100 \mu \mathrm{L}$ of each diluted sample was plated on PDA (potato $200 \mathrm{~g} / \mathrm{L}$, sucrose $10 \mathrm{~g} / \mathrm{L}$, sea salt $30 \mathrm{~g} / \mathrm{L}, 20 \mathrm{~g} / \mathrm{L}$ agar), Gause's NO. 1 (soluble starch $20 \mathrm{~g} / \mathrm{L}, \mathrm{NaCl} 0.5 \mathrm{~g} / \mathrm{L}, \mathrm{K}_{2} \mathrm{HPO}_{4} 0.5 \mathrm{~g} / \mathrm{L}, \mathrm{FeSO}_{4} 0.01 \mathrm{~g} / \mathrm{L}$, $\mathrm{MgSO}_{4} \cdot 7 \mathrm{H}_{2} \mathrm{O} 0.5 \mathrm{~g} / \mathrm{L}, \mathrm{KNO}_{3} 1 \mathrm{~g} / \mathrm{L}$, sea salt $30 \mathrm{~g} / \mathrm{L}, 20 \mathrm{~g} / \mathrm{L}$ agar, $\mathrm{pH}$ 7.2-7.4) and MM medium (yeast extract $3 \mathrm{~g} / \mathrm{L}$, peptone $5 \mathrm{~g} / \mathrm{L}$, sea salt $30 \mathrm{~g} / \mathrm{L}, 20 \mathrm{~g} / \mathrm{L}$ agar, $\mathrm{pH}$ 7.2-7.4) and separately incubated at either $28^{\circ} \mathrm{C}$ or $25^{\circ} \mathrm{C}$ for $2-7$ days as necessary. To make broth medium, agar was absent. The isolated colonies were purified by streak-plate technique. Then, each pure isolate was cultured in $50 \mathrm{~mL}$ of broth medium and culture supernatant was collected after centrifuge at $8000 \mathrm{rpm}$ for $10 \mathrm{~min}$ at $4^{\circ} \mathrm{C}$ for subsequent LAAO activity screening.

\subsection{Screening of LAAO-Producing Microorganism. LAAO} activity produced by isolate was detected by measuring its oxidization productions of $\mathrm{L}$-amino acids including $\mathrm{H}_{2} \mathrm{O}_{2}$, aketo acid, and $\mathrm{NH}_{4}{ }^{+}$. (1) $\mathrm{H}_{2} \mathrm{O}_{2}$ measurement: $1.5 \mathrm{~mL}$ of resultant culture supernatant was mixed with $1.5 \mathrm{~mL}$ of $10 \mathrm{mM} \mathrm{L}$ Leu for oxidization reaction at $37^{\circ} \mathrm{C}$ for $30 \mathrm{~min}$. Then, the produced $\mathrm{H}_{2} \mathrm{O}_{2}$ was measured using Amplex Red Hydrogen Peroxide/Peroxidase Assay kit (Invitrogen, USA) according to manufacture's instruction. (2) a-Keto acid measurement: $1.5 \mathrm{~mL}$ of culture supernatant was mixed with $1.5 \mathrm{~mL}$ of $10 \mathrm{mM}$ $\mathrm{L}$-Leu for oxidization reaction at $37^{\circ} \mathrm{C}$ for $30 \mathrm{~min}$. Then, as reported [13], the produced a-keto leucine was detected using 2,4-dinitrophenylhydrazine (DNP) which can react with carbonyl group to generate brown-red dinitrophenylhydrazone. Briefly, $550 \mu \mathrm{L}$ of detection solution was mixed with $450 \mu \mathrm{L}$ of $20 \%$ trichloroacetic acid and kept at room temperature for $30 \mathrm{~min}$. Next, $200 \mu \mathrm{L}$ of $20 \mathrm{mM}$ DNP was added and the mixture was incubated at room temperature for $15 \mathrm{~min}$. The reaction was terminated by adding $4 \mathrm{~mL}$ of $0.8 \mathrm{M} \mathrm{NaOH}$. Finally, the mixture was centrifuged at $12,000 \mathrm{rpm}$ for $10 \mathrm{~min}$ and the absorbance of the supernatant was measured at
$520 \mathrm{~nm}$. (3) $\mathrm{NH}_{4}{ }^{+}$detection: $25 \mathrm{~mL}$ of resultant culture supernatant was mixed with $25 \mathrm{~mL}$ of $10 \mathrm{mM} \mathrm{L}$-Leu in $250 \mathrm{~mL}$ glass flask for oxidization reaction at $37^{\circ} \mathrm{C}$ for $30 \mathrm{~min}$. Then, $1 \mathrm{~mL}$ of $1 \mathrm{M} \mathrm{NaOH}$ was added and paper $\mathrm{pH}$ indicator was put above the glass flask. After shaking at $120 \mathrm{rpm}$ for $60 \mathrm{~min}$ at room temperature, the color change of paper $\mathrm{pH}$ indicator due to the $\mathrm{NH}_{3}$ release was observed.

2.3. Molecular Taxonomy and Phylogenetic Analysis. The genomic DNA of LAAO-producing microorganism was extracted by bacterial DNA isolation method [14]. 16S rDNA gene was amplified in $50 \mu \mathrm{L}$ containing $37 \mu \mathrm{L}$ of $\mathrm{ddH}_{2} \mathrm{O}$, $5 \mu \mathrm{L}$ of $10 \times$ Easy Taq buffer, $4 \mu \mathrm{L}$ of $2.5 \mathrm{mM}$ dNTPs, $100 \mathrm{nM}$ forward primer 27F ( $5^{\prime}$-GAGTTTGATCCTGGCTCAG-3'), $100 \mathrm{nM}$ reverse primer 1527R (5'-AGAAAGGAGGTGATCCAGCC- $3^{\prime}$ ), 1 ng genomic DNA, and $1 \mathrm{U}$ Taq DNA polymerase with denaturation at $94^{\circ} \mathrm{C}$ for $5 \mathrm{~min}$ followed by 30 cycles of $1 \mathrm{~min}$ at $94^{\circ} \mathrm{C}, 50 \mathrm{~s}$ at $55^{\circ} \mathrm{C}, 90 \mathrm{~s}$ at $72^{\circ} \mathrm{C}$, and a final 10 min extension at $72^{\circ} \mathrm{C}$. At the end of reaction, $\mathrm{PCR}$ product was cooled to $4^{\circ} \mathrm{C}$ to await further use. After size confirmation on $1.0 \%$ agarose gel, the PCR product was sent to Sangon Biotech (Shanghai) Co. Ltd for sequencing of $16 \mathrm{~S}$ rDNA. The similarity and homology of $16 \mathrm{~S}$ rDNA gene sequence was analyzed using BLAST search available in genbank of NCBI. The DNA sequences were aligned and phylogenetic tree was constructed by neighbor joining method with bootstrap trials 1000 using MegaV4.0.2 software.

2.4. Physiological and Biochemical Characterization. The ability of the isolate to utilize various carbon and nitrogen sources and other physiological and biochemical properties was studied according to the recommendation in "The Manual of Systematic Methods of Determinative Bacterial.”

2.5. Retrieval of LAAO Gene. To retrieve the full length of laogene sequence from isolated microbial producer, two rounds of PCR were performed. For the first round PCR, the 6 degenerate primers were designed based on the conserved regions after alignment of LAAO sequences from general LAAOproducing marine microorganisms (see Table 1) including Comamonas testosteroni KF-1 (ZP_03541004), Dinoroseobacter shibae DFL 12 (ABV95616), and Caulobacter sp. K31 (YP_001683007) and LAAO-producing Pseudoalteromonas microorganisms (see Table 2) including Pseudoalteromonas haloplanktis TAC125 (YP_339251), Pseudoalteromonas tunicata D2 (ZP_01132853), Pseudoalteromonas atlantica T6c (YP_662716), and Pseudoalteromonas sp. SM9913 (YP_004069407). ZZ-1, ZZ-2 and ZZ-3 were forward primers and $\mathrm{ZF}-1, \mathrm{ZF}-2$, and $\mathrm{ZF}-3$ are reverse ones. As required, these 6 degenerate primers combined with each other to form 9 sets of forward and reverse primer pairs. PCR amplification was performed with genomic DNA of LAAOproducer as template and PrimeSTAR HS DNA polymerase (TaKaRa, Dalian, China) as polymerase. The Touch-down PCR consisted of denaturation at $94^{\circ} \mathrm{C}$ for $5 \mathrm{~min}, 15$ cycle of $30 \mathrm{~s}$ at $94^{\circ} \mathrm{C}, 30 \mathrm{~s}$ at $64^{\circ} \mathrm{C}\left(-1^{\circ} \mathrm{C} /\right.$ cycle $)$, and $1 \mathrm{~min}$ at $72^{\circ} \mathrm{C}$, followed by another 20 cycles of $30 \mathrm{~s}$ at $94^{\circ} \mathrm{C}, 30 \mathrm{~s}$ at $48^{\circ} \mathrm{C}$, and $1 \mathrm{~min}$ at $72^{\circ} \mathrm{C}$ and a final $10 \mathrm{~min}$ extension at $72^{\circ} \mathrm{C}$. After size 
TABLE 1: Conserved regions of LAAOs from common marine microorganisms used for design of degenerate primers.

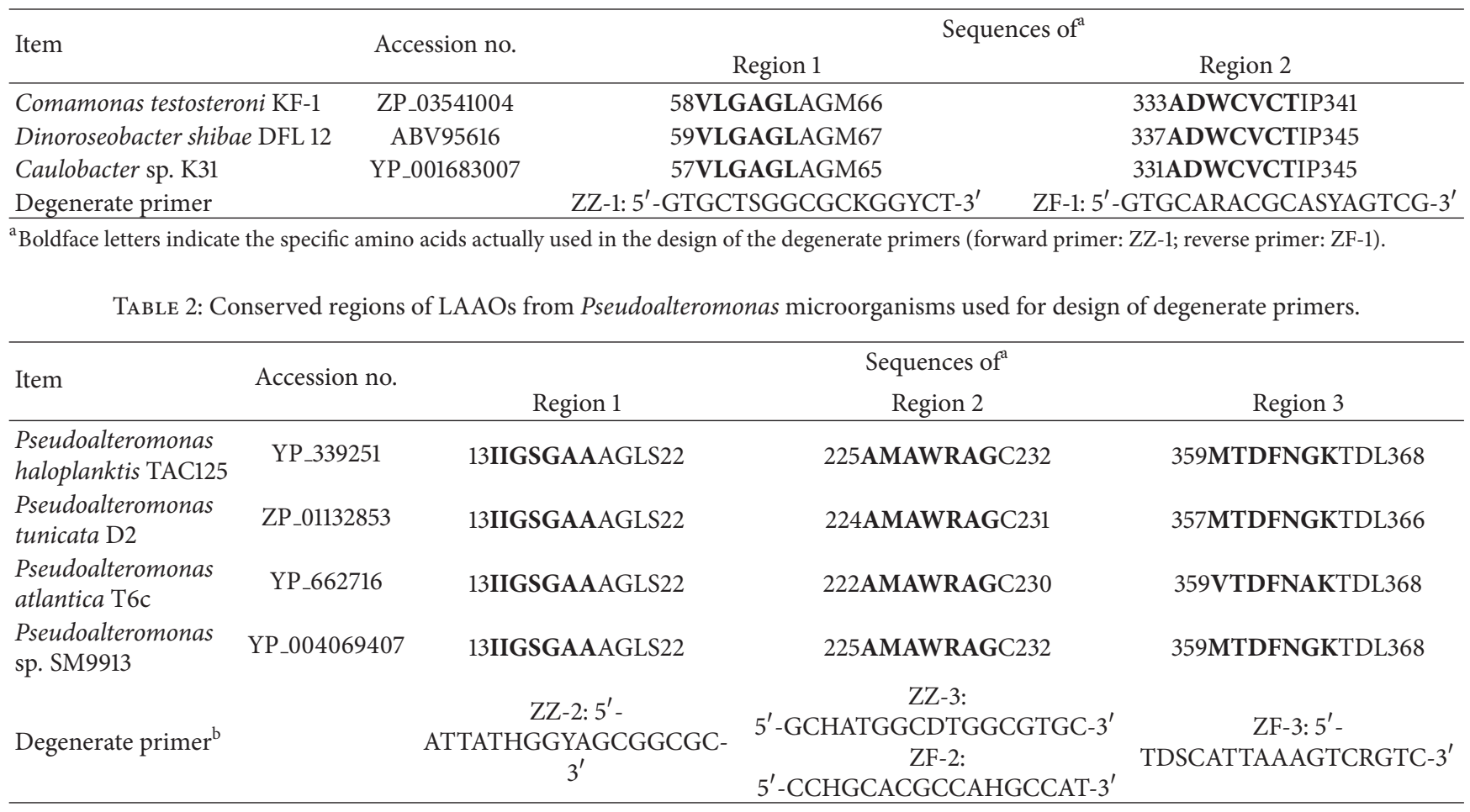

${ }^{a}$ Boldface letters indicate the specific amino acids actually used in the design of the degenerate primers (forward primers: ZZ-2 and ZZ-3; reverse primers: ZF2 and ZF-3).

TABLE 3: Primers used in cloning of $5^{\prime}$ and $3^{\prime}$ flanked sequences of LAAO gene from Pseudoalteromonas sp. B3.

\begin{tabular}{|c|c|c|}
\hline Primers* & Sequences $\left(5^{\prime}-3^{\prime}\right)$ & Size (bp) \\
\hline \multicolumn{3}{|l|}{ Set A } \\
\hline $5 \mathrm{~F}-1$ & TTGCCAAAGACAGAGCCAGGCT & 22 \\
\hline $5 \mathrm{~F}-2$ & AGGTCGCCCTTACTGAGCACTGT & 23 \\
\hline $5 \mathrm{~F}-3$ & TCTTATCAAACACCGCAGCAAT & 22 \\
\hline \multicolumn{3}{|l|}{ Set B } \\
\hline 3Z-1 & ATTGCCATGGCTTGGCGTG & 18 \\
\hline $3 Z-2$ & GTCAAATCCAGACGTTTCCAGT & 22 \\
\hline $3 Z-3$ & GCGGTGCCAGTAAAGTGTATCT & 22 \\
\hline \multicolumn{3}{|l|}{ Set C } \\
\hline S-1 & ACGATGGACTCCAGAGCGGCCCGCVNVNNNGGAA & 34 \\
\hline S-2 & ACGATGGACTCCAGAGCGGCCCGCBNBNNNGGTT & 34 \\
\hline S-3 & ACGATGGACTCCAGAGCGGCCCGCHNVNNNCCAC & 34 \\
\hline S-4 & ACGATGGACTCCAGAGCGGCCCGCVVNVNNNCCAA & 35 \\
\hline S-5 & ACGATGGACTCCAGAGCGGCCCGCBDNBNNNCGGT & 35 \\
\hline B0043 & NNNNNN & 6 \\
\hline B0043-9 & NNNNNNNNN & 9 \\
\hline B0043-10 & NNNNNNNNNN & 10 \\
\hline
\end{tabular}

${ }^{*}$ Primers in set A are one set of nested specific primers for amplification of $5^{\prime}$ flanked region of B3-LAAO gene; primers in set B are one set of nested primers for amplification of $3^{\prime}$ flanked region of B3-LAAO gene; primers in set $\mathrm{C}$ are arbitrary primers for amplification of $5^{\prime}$ and $3^{\prime}$ flanked regions of B3-LAAO gene.

confirmation on $1 \%$ agarose gel and gel extraction (Qiagen, CA, USA), the PCR product with expected size was cloned into pMD19-T simple vector (TaKaRa, Dalian, China) and sequenced by Sangon Biotech (Shanghai) Co. Ltd.

To obtain the entire lao-gene, the second round of inverse PCR (using nested-PCR technique) was employed to amplify the flanked gene sequences $\left(5^{\prime}\right.$ and $3^{\prime}$ regions). As shown in Table 3, two nested specific primer sets, $5 \mathrm{~F}-1$ (1st), $5 \mathrm{~F}-2$ (2nd), and 5F-3 (3rd) for $5^{\prime}$ flanked region and 3Z-1 (1st), 3Z2 (2nd), and $3 Z-3$ (3rd) for $3^{\prime}$ flanked region, were designed based on the intermediated lao-gene sequence obtained in the first round of PCR. Nine arbitrary primers (S-1, S-2, S-3, S-4, 
S-5, S-6, B0043, B0043-9, and B0043-10) (see Table 3) were purchased from Invitrogen (USA) and each arbitrary primer was used to pair with nested specific primer to form forward and reverse primer set. For either $5^{\prime}$ or $3^{\prime}$ flanked region of lao-gene, a total of 27 nested-PCR amplification reactions were performed with denaturation at $94^{\circ} \mathrm{C}$ for $5 \mathrm{~min}, 6$ cycles of $30 \mathrm{~s}$ at $94^{\circ} \mathrm{C}, 50 \mathrm{~s}$ at $35^{\circ} \mathrm{C}$, and $2 \mathrm{~min}$ at $72^{\circ} \mathrm{C}$, followed by another 30 cycles of $60 \mathrm{~s}$ at $94^{\circ} \mathrm{C}, 50 \mathrm{~s}$ at $55^{\circ} \mathrm{C}$, and $2 \mathrm{~min}$ at $72^{\circ} \mathrm{C}$ and a final $10 \mathrm{~min}$ extension at $72^{\circ} \mathrm{C}$. The desired PCR product was purified using a gel extraction kit (Qiagen, CA, USA) and cloned into pMD19-T simple vector (TaKaRa, Dalian, China). After sequencing by Sangon Biotech (Shanghai) Co. Ltd, the contigs of $5^{\prime}$ flanked fragment, intermediated fragment, and $3^{\prime}$ flanked fragment were assembled using "Cap Conting Assembly" of BioEdit software V5.2.

2.6. Cloning and Expression of LAAO in E. coli BL21(DE3). The complete lao-gene was amplified using a pair of forward primer (B3laaoF, 5 '-CGCGGATCCTATGAAAGAACAAGTTC- $3^{\prime}$, with a BamH I restriction site as underlined) and reverse primer (B3laaoR, $5^{\prime}$-CCCAAGCTTACGTTTGATTTTACTGG-3', with a Hind III restriction site). $P f u$ DNA polymerase (Promega) was used in a PCR to amplify the 1,608 bps lao-gene. The desired PCR product was subsequently cloned into pMD19-T simple vector, and the lao-gene sequence was confirmed by BamH I and Hind III digestion and direct DNA sequencing. After BamH I-Hind III digestion, the released lao-gene was subsequently cloned into the BamH I-Hind III restriction sites of expression vectors, pET-28b $(+)$ and pET-20b(+) (Novagen, USA), to generate pET-28b(+)-lao and pET-20b(+)-lao, respectively. The inserted gene was confirmed by DNA sequencing (Sangon Biotech (Shanghai) Co. Ltd.). The plasmids, pET-28b(+)lao and pET-20b(+)-lao, inserted with a PCR-amplified laogene was separately transformed into E. coli BL21(DE3) (Novagen) cells and cultured in LB medium supplemented with $50 \mu \mathrm{g} / \mathrm{mL}$ kanamycin and $100 \mu \mathrm{g} / \mathrm{mL}$ ampicillin. Cells were grown at $37^{\circ} \mathrm{C}$ to an $\mathrm{OD}_{600}$ of 0.4 to 0.6 . Overproduction of the LAAO protein was induced by addition of $1 \mathrm{mM}$ isopropyl- $\beta$-D-thiogalactopyranoside (IPTG) and $0.1 \mathrm{mM}$ FAD together with $0.1 \mathrm{mM} \mathrm{Zn}^{2+}$ at $25^{\circ} \mathrm{C}$ for $6 \mathrm{~h}$.

After expression, the cells from $1.5 \mathrm{~mL}$ of culture were harvested by a centrifugation at $6,000 \mathrm{rpm}$ for $5 \mathrm{~min}$ at $4^{\circ} \mathrm{C}$. Then, the cell pellets were resuspended in $100 \mu \mathrm{L}$ of $\mathrm{ddH}_{2} \mathrm{O}$ and mixed with $25 \mu \mathrm{L}$ of 4 -fold sample loading buffer $(1.0 \mathrm{M}$ Tris-HCl, pH 6.8, 10\% SDS, $20 \% \beta$-mercaptoethanol, $50 \%$ glycerol, $1 \%$ bromophenol blue). After boiling for $5 \mathrm{~min}$, $30 \mu \mathrm{L}$ of resultant sample mixture was subjected to SDSpolyacrylamide gel (SDS-PAGE) with a stacking gel of $5 \%$ and separation gel of $12 \%$, as described [15], and the number and molecular weight of the expressed LAAO were determined.

2.7. Assay of the Expressed LAAO Activity. The expressed LAAO activity was determined by measuring the produced $\mathrm{H}_{2} \mathrm{O}_{2}$ with Prussian blue agar assay [16]. In brief, $25 \mathrm{~mL}$ of the induced cell culture was mixed with $25 \mathrm{~mL}$ of $10 \mathrm{mM} \mathrm{L}$ Leu for oxidation reaction at $37^{\circ} \mathrm{C}$ with shaking at $120 \mathrm{rpm}$. After incubation for $1 \mathrm{~h}, 50 \mu \mathrm{L}$ of resultant oxidation mixture
TABle 4: Detection of the $\mathrm{H}_{2} \mathrm{O}_{2}$ production using Amplex Red Hydrogen Peroxide/Peroxidase Assay kit.

\begin{tabular}{lcc}
\hline Reactions & Amount of $\mathrm{H}_{2} \mathrm{O}_{2}(\mathrm{mM})$ & Relative activity (\%) \\
\hline Test $\mathrm{1}^{\mathrm{a}}$ & 1.84 & 100 \\
Test $2^{\mathrm{b}}$ & 0.14 & 7.61 \\
Test $3^{\mathrm{c}}$ & 0.043 & 2.34 \\
\hline
\end{tabular}

${ }^{\mathrm{a} C u l t u r e}$ supernatant of strain B3 with substrate L-Leu; ${ }^{\mathrm{b}}$ culture supernatant of strain B3 without substrate L-Leu; ${ }^{\mathrm{c}}$ same as Test 1, except that the culture supernatant of strain B3 was denatured with boiling before addition of substrate L-Leu.

was subjected to the circular well with diameter of $6 \mathrm{~mm}$ on Prussian blue agar $\left(1.0 \mathrm{~g} / \mathrm{L} \mathrm{FeCl} 3 \cdot 6 \mathrm{H}_{2} \mathrm{O}, 1.0 \mathrm{~g} / \mathrm{L}\right.$ potassium hexacyanoferrate (III), 2\% agar, $\mathrm{pH}$ 7.5). After incubation for $30 \mathrm{~min}$ at room temperature, the color development resulted from Prussian blue formation due to the $\mathrm{H}_{2} \mathrm{O}_{2}$ production was monitored.

\section{Results and Discussion}

3.1. Isolation of LAAO-Producing Strain. Based on morphological identification, a total of 157 pure isolates, 32 from PDA medium, 51 from Gause's NO. 1 medium, and 74 from MM medium, were obtained. Out of those 157 isolates subjected to LAAO-producing screening, only one isolate (strain B3) gave the capability to produce LAAO, as determined and verified below.

First, LAAOs are able to catalyze L-amino acids to release $\mathrm{H}_{2} \mathrm{O}_{2}$ which can be detected using Amplex Red Hydrogen Peroxide/Peroxidase Assay kit. As shown in Table 4, almost no $\mathrm{H}_{2} \mathrm{O}_{2}$ was detected from either the culture supernatant of strain B3 without L-Leu (Test 2) or the denatured culture supernatant by boiling for 10 minutes followed by addition of L-Leu (Test 3). In contrast, decent amount of $\mathrm{H}_{2} \mathrm{O}_{2}$ $(1.84 \mathrm{mM})$ was measured from the culture supernatant of strain B3 with L-Leu (Test 1), meaning that the culture supernatant of strain $\mathrm{B} 3$ can use L-Leu to release $\mathrm{H}_{2} \mathrm{O}_{2}$. All these findings indicate that strain $\mathrm{B} 3$ may bear the ability to produce LAAO. Second, as carbonyl derivative, a-keto acid can react with 2,4-dinitrophenylhydrazine (DNP) to generate brown-red dinitrophenylhydrazone with maximum absorbance at $520 \mathrm{~nm}$ [13]. As shown in Figure 1, the culture supernatant of strain $\mathrm{B} 3$ without L-Leu gave flaxen and $\mathrm{OD}_{520}$ was low, displaying the similar result as the denatured culture supernatant of strain B3 with L-Leu. In contrast, both the aketo leucine (positive control) and the oxidation solution of L-Leu by the culture supernatant of strain B3 had brown-red color with high $\mathrm{OD}_{520}$ value. All these findings indicate that L-Leu can be oxidized by culture supernatant of strain B3 to generate the corresponding a-keto acid. Therefore, strain B3 can produce LAAO. Third, in the presence of strong base like $\mathrm{NaOH}, \mathrm{NH}_{4}{ }^{+}$in solution will become unstable to release $\mathrm{NH}_{3}$ which can easily be detected by paper $\mathrm{pH}$ indicator. The results in Figure 2 showed that after addition of $\mathrm{NaOH}$, the culture supernatant of strain B3 without L-Leu (negative control) did not cause the color change of the paper $\mathrm{pH}$ indicator, maintaining the original yellow. In contrast, like the 


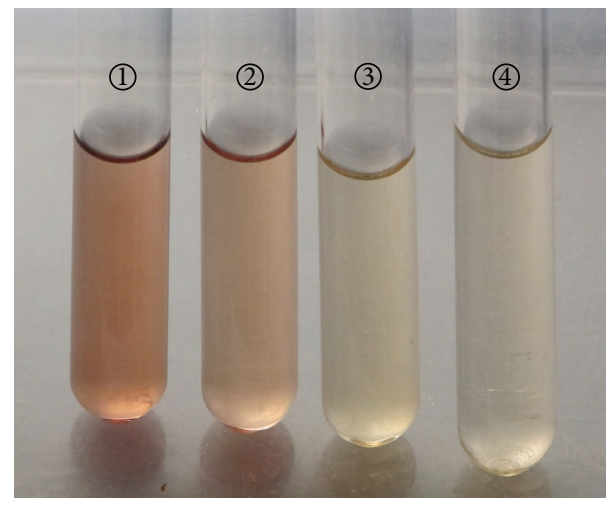

FIgURE 1: Detection of a-keto acid production using 2,4dinitrophenylhydrazine (DNP) [13]. (1) positive control: $\alpha$-keto leucine in MM medium (OD520 = 0.232); (2) culture supernatant of strain B3 with substrate L-Leu (OD520 = 0.198); (3) culture supernatant of strain B3 without substrate L-Leu (OD520 = 0.032); (4) boiling-denatured culture supernatant of strain B3 with substrate L-Leu $($ OD520 = 0.045).

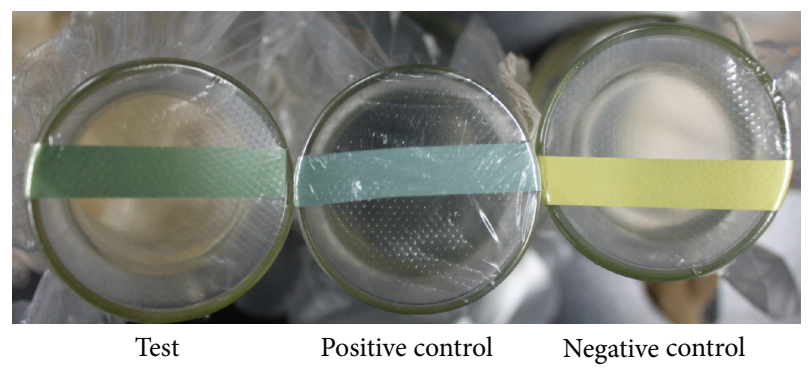

Figure 2: Determination of the presence of $\mathrm{NH}_{4}{ }^{+}$after addition of $\mathrm{NaOH}$ using paper $\mathrm{pH}$ indicator. Test: culture supernatant of strain B3 with substrate L-Leu; positive control: ammonia solution; negative control: culture supernatant of strain B3 without substrate L-Leu.

positive control (ammonia solution), the oxidization solution of L-Leu by the culture supernatant of strain B3 caused the color change of paper $\mathrm{pH}$ indicator from original yellow to absinthe-green. All these findings indicate that L-Leu can be used by the culture supernatant of strain B3 to release $\mathrm{NH}_{4}{ }^{+}$, supporting that strain B3 can produce LAAO.

Our quantitative and qualitative measurements together clearly showed that the culture supernatant of strain B3 can specifically oxidize L-Leu to yield $\mathrm{H}_{2} \mathrm{O}_{2}$, a-keto leucine, and $\mathrm{NH}_{4}{ }^{+}$, thus crossly supporting that the isolate $\mathrm{B} 3$ can produce LAAO. Among these three detection methods, $\mathrm{H}_{2} \mathrm{O}_{2}$ assay kit and DNP assay are quantitative methods with very high sensitivity, whereas paper $\mathrm{pH}$ indicator can only be used for qualitative measurement with low sensitivity. On the other hand, compared with the former two methods, the latter one gives advantages including convenience, no requirement of instrument, and cost-effectiveness, thus ideal for the first-step screening of LAAO-producing microorganisms.

3.2. Brief Characterization of Strain B3. A pair of primers, $27 \mathrm{~F}$ and $1527 \mathrm{R}$, were successfully used to amplify about $1.5 \mathrm{~kb}$
$16 \mathrm{~S}$ rDNA gene from strain B3. After sequencing, a total of $1396 \mathrm{bp}$ were gained. The Blast analysis of $16 \mathrm{~S} \mathrm{rDNA}$ at the NCBI website together with the constructed phylogenetic tree (see Figure 3) indicated that strain B3 was very proximal to Pseudoalteromonas spp. with the highest identity of $98.5 \%$ to Pseudoalteromonas viridis. On MM medium plate, strain B3 exhibited yellow smooth round. The Gram-staining reaction showed that it was Gram-negative (photo not shown). The physiological and biochemical analysis revealed that it was positive to starch hydrolysis, indole test, citrate utilization, and oxidase test and negative to lysine decarboxylation enzyme, half a solid agar, gelatin hydrolysis, $\mathrm{H}_{2} \mathrm{~S}$ production, acetamide, V-P test, methyl red test, and melezitose monohydrate. In addition, it can consume galactose, glucose, lactose, fructose, maltose, and rhamnose as carbon source and $\left(\mathrm{NH}_{4}\right)_{2} \mathrm{HPO}_{4}, \mathrm{KNO}_{3}$, arginine, methionine, glycine, and tyrosine as nitrogen sources for growth. Therefore, it was designated as Pseudoalteromonas sp. B3.

3.3. Retrieval of lao Gene and Characterization of the Deduced Amino Acid Sequence. Among 9 sets of primer pairs, only one pair from ZZ-2 and ZF-2 gave the desired PCR-amplified product with around $650 \mathrm{bps}$ in size in the first round of PCR. After sequencing, 654 bps of intermediated LAAO-coding sequence from Pseudoalteromonas sp. B3 was obtained to show $80 \%$ identity to L-aspartate oxidase-coding gene from Pseudoalteromonas sp. SM9913. In the second round of inverse PCR, only arbitrary primer S-2 was combined with one set of nested specific primers (5F-1, 5F-2, and 5F-3) to amplify the desired $5^{\prime}$ flanked region. Similarly, only arbitrary primer BOO43 was successfully used to pair with the other set of nested specific primers (3Z-1, 3Z-2, and 3Z$3)$ to amplify the $3^{\prime}$ flanked region. After sequence assembly, a full B3-LAAO-coding sequence with 1608 bps in length was obtained. This open reading frame (ORF) encodes 535 amino acid (AA) residues with a predicted molecular mass of $59571.46 \mathrm{Da}(60 \mathrm{kDa})$. A BLASTN search of the ORF nucleotide sequence at the NCBI website showed that it shared $75 \%$ and $73 \%$ identity to L-aspartate oxidase from Pseudoalteromonas sp. SM9913 and Shewanella denitrificans OS217, respectively. According to the deduced amino acid sequence, further BLASTP search at the NCBI website revealed that B3-LAAO had the highest homology with a number of L-aspartate oxidases, giving $80 \%, 80 \%, 80 \%$, $73 \%$, and $70 \%$ identity to L-aspartate oxidases from Pseudoalteromonas sp. SM9913, Pseudoalteromonas haloplanktis ANT/505, Pseudoalteromonas tunicata D2, Alishewanella jeotgali KCTC 22429, and Shewanella denitrificans OS217, respectively. So far, most of LAAOs found in Pseudoalteromonas genus have a strict preference for specific substrate, for example, L-aspartate oxidase from Pseudoalteromonas sp. SM9913, Pseudoalteromonas haloplanktis ANT/505 and Pseudoalteromonas tunicata D2, and L-lysine oxidase from Pseudoalteromonas tunicate D2 [17]. In contrast, B3-LAAO exhibited a broad substrate spectra with the highest activity to L-Leu, followed by L-Lys, L-Tys, L-Asn, L-Gln, L-Met, Lcystine, L-Arg, L-Trp, and L-Glu but no detectable activity to L-aspartic acid (data not shown). So that B3-LAAO 


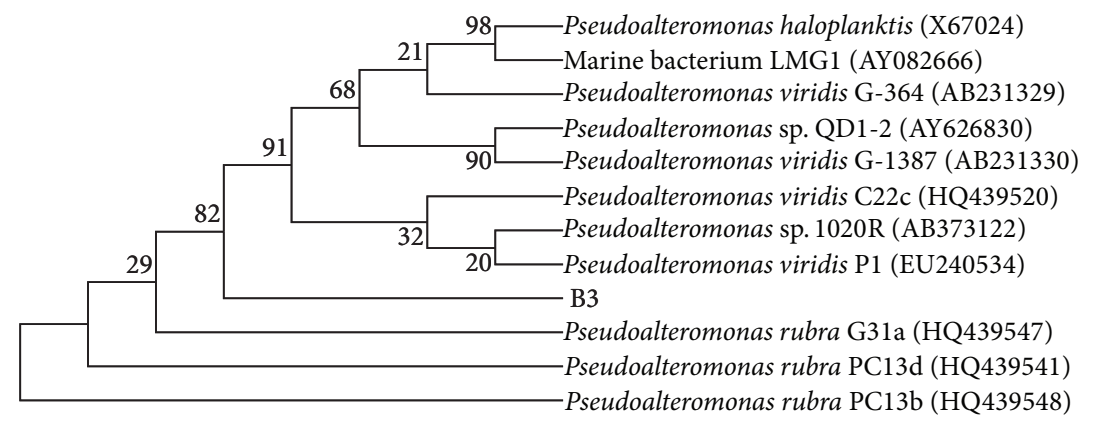

FIgure 3: Phylogenetic tree of strain B3 based on $16 \mathrm{~S}$ rDNA. The numbers at the nodes indicate the bootstrap level based on the analysis of 1000 resampled data sets.

Consensus
Pseudoalteromonas sp. B3
Pseudoalteromonas sp. SM9913
Pseudoalteromonas haloplanktis ANT/505
Pseudoalteromonas tunicata D2
Alishewanella jeotgali KCTC 22429
Shewanella denitrificans OS217

Figure 4: Sequence alignment between B3-LAAO and several homologous LAAOs. The highly conserved motifs, dinucleotide-binding domain motif ohhhhGxGxxGxxxhxxL for FAD binding where o stands for a polar or charged residue and h for a hydrophobic residue, and characteristic motif RxGG, are indicated.

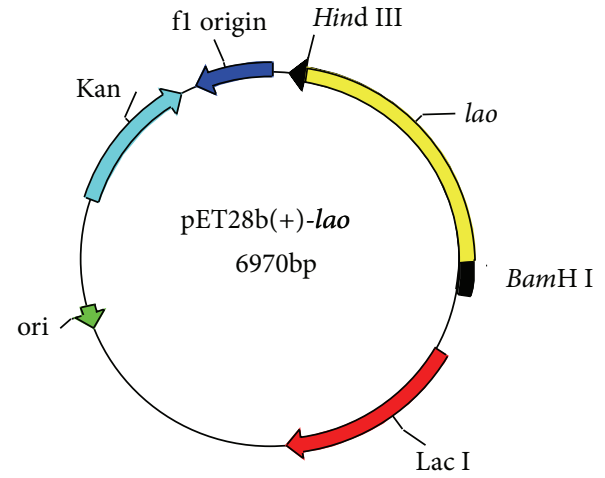

(a)

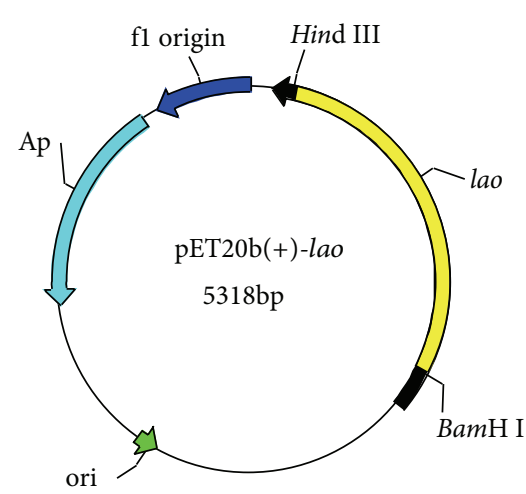

(b)

FIGURE 5: Maps of the recombinant plasmids containing lao-gene, pET28b(+)-lao (a), and pET20b(+)-lao (b). The LAAO-encoding gene was cloned between BamH I and Hind III restriction sites.

could represent a novel LAAO, which can certainly broaden our view on LAAO from Pseudoalteromonas genus. Based on the alignment of amino acid sequences of 6 homologous LAAOs (see Figure 4), B3-LAAO gave two highly conserved motifs. One is the dinucleotide-binding motif ohhhhGxGxxGxxxhxxL which is a typical FAD binding site where o stands for a polar or charged residue and $h$ for a hydrophobic residue; the other is the characteristic sequence motif RxGG. These motifs appear in several families of the flavoproteins $[1,8]$.

3.4. Expression of B3-LAAO in E. coli BL2 (DE3). Attempts to express B3-LAAO in E. coli were made and two different expression vectors pET-28b(+)-lao and pET-20b(+)-lao were constructed for this purpose (see Figure 5). The laogene was cloned into pET28b(+) and pET20b(+), respectively, and both were separately transformed into $E$. coli BL21(DE3) strain and expressed as described in Section 2. PCR amplification, restriction endonuclease digestion, and direct sequencing analysis demonstrated that plasmids of pET28b(+)-lao and pET20b(+)-lao were successfully constructed (data not shown). SDS-PAGE analysis (see Figure 6) showed that the recombinant B3-LAAOs on the two different vectors both can be induced and expressed to give the same molecular mass of about $60 \mathrm{kDa}$, agreeing with the calculated one based on the deduced amino acid sequence.

The activity of the expressed recombinant LAAO was determined with Prussian blue agar assay [16] using L-Leu 


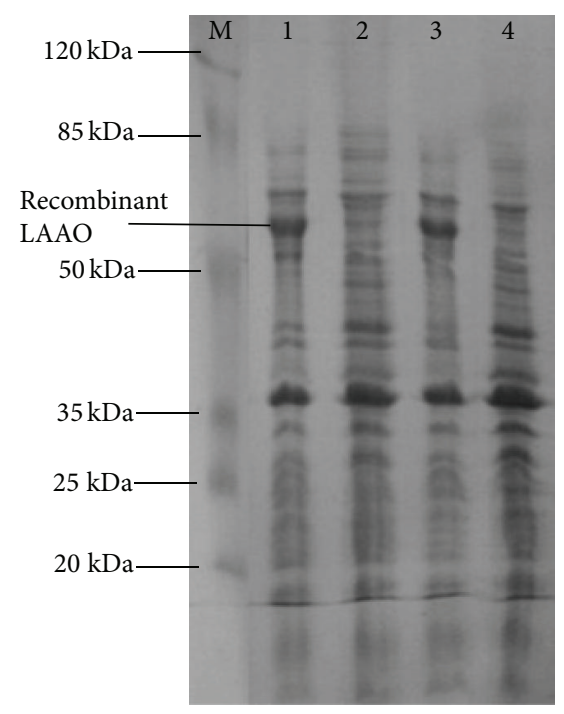

FIGURE 6: SDS-PAGE analysis of the expressed recombinant B3LAAO in E. coli BL21(DE3). Lane M: prestained protein marker (Thermo, USA); lane 1: cell culture of E. coli BL21(DE3)/pET28b(+)lao with a 6 h-induction by IPTG; lane 2: cell culture of $E$. coli BL21(DE3)/pET28b(+)-lao without induction; lane 3: cell culture of $E$. coli BL21(DE3)/pET20b(+)-lao with a 6 h-induction by IPTG; Lane 4: cell culture of E. coli BL21(DE3)/pET20b(+)-lao without induction.

as substrate. The results in Figure 7 indicated that, after induction by IPTG, the recombinant B3-LAAOs in culture supernatants from $E$. coli BL21(DE3) transformed with both pET28b(+)-lao and pET20b(+)-lao can cause the Prussian blue agar to form blue halos which resulted from the $\mathrm{H}_{2} \mathrm{O}_{2}$ production, while no LAAO activity could be detected in all tested samples from the sonicated cell pellets (data not shown). In fact, accumulation of inclusion bodies was found in cell pellets. These inclusion bodies could be dissolved in $8 \mathrm{M}$ urea, but the enzyme activity could not be restored by slowly diluting the solution. All these findings indicate that B3-LAAO could functionally be expressed in E. coli, but the expressed level was relatively low. The following reasons may account for this phenomenon. First, $\mathrm{H}_{2} \mathrm{O}_{2}$ produced by LAAO activity is toxic to $E$. coli [18], and thus this would likely inhibit the LAAO expression level; second, the recombinant LAAO from $E$. coli may be much less active than the wild-type one; third, E. coli may be a relatively unsuitable host for the heterologous production of B3-LAAO. Several ways may give solutions to these common problems. First, other hosts can be used to replace E. coli for heterologous expression of LAAO [9]. Second, coexpression of chaperone protein may prevent the aggregation of the recombinant LAAO in E. coli [19]. Third, the expression conditions can be varied, considering the inductor concentration, time of induction, induction temperature, and duration, to reduce the amount of insoluble fractions. Last but not least, inclusion bodies can be dissolved into urea for restoration of its activity through dialysis.

It has been found that the change in diameter of blue halo on Prussian blue agar is a function of $\mathrm{H}_{2} \mathrm{O}_{2}$ concentration with exponential fit [16]. According to the size of halos, Figure 7 further showed that E. coli BL21(DE3)/pET20b(+)lao with a signal peptide had the higher activity than $E$. coli BL21(DE3)/pET28b(+)-lao without signal peptide. Most probably, secretion of recombinant LAAO can help to reduce the harm of intracellular active LAAO to cells.

\section{Conclusion}

In the present study, an LAAO-producing marine bacterium B3 was successfully isolated from the intertidal sea area. $16 \mathrm{~S}$ rDNA sequence and phylogenetic tree analysis together with physiological and biochemical assays revealed that it can be designated as Pseudoalteromonas sp. B3. Its capability of LAAO production was demonstrated by determining the release of $\mathrm{H}_{2} \mathrm{O}_{2}, \alpha$-keto leucine, and $\mathrm{NH}_{4}{ }^{+}$after oxidization of L-Leu. Through two rounds of PCR, entire LAAO-coding gene sequence from Pseudoalteromonas sp. B3 was obtained. Its deduced amino acid sequence of 535 residues showed two conserved motifs, GG-motif and $\beta \alpha \beta$-dinucleotide-binding domain motif, typical to a number of flavoproteins. B3LAAO had the highest identity of only around $80 \%$ to several L-aspartate oxidases from different microorganisms. In addition, it had high activities to broad L-amino acids but not to L-aspartic acid. Therefore, B3-LAAO probably presents a novel LAAO, which can enrich our knowledge on LAAO from Pseudoalteromonas genus.

To date, heterologous expression of LAAO is still a big challenge due to its toxicity to host and the requirement of posttranslational modification. In this study, B3-LAAO was successfully and functionally expressed in E. coli BL21(DE3) using two different vectors pET28b(+) and pET20b(+). It was found that addition of suitable concentration FAD and $\mathrm{Zn}^{2+}$ during IPTG induction can increase the activity of recombinant B3-LAAO. Besides, lower induction temperature $\left(25^{\circ} \mathrm{C}\right)$ was better for B3-LAAO expression than higher induction temperature $\left(37^{\circ} \mathrm{C}\right)$. Most probably, low temperature can reduce the aggregation of $\mathrm{B} 3$-LAAO. In future, further lower induction temperature $\left(16^{\circ} \mathrm{C}\right)$ is highly promising. No active intracellular LAAO activity was detected, but huge inclusion bodies were formed in cell, which is common in heterologous expression of LAAO $[9,11]$. Overall, the expression level of active B3-LAAO was relatively low. We believe that this level is only for soluble LAAO, while much of the LAAO is present in insoluble inclusion bodies. Therefore, in future, the expression conditions will be optimized, considering the inductor concentration, time of induction, induction temperature, and duration. In addition, the inclusion bodies will be dissolved into urea for its activity restoration. Finally, coexpression of chaperone protein with B3-LAAO in E. coli is also being performed in our lab.

\section{Conflict of Interests}

The authors have declared that no conflict of interests exists. 

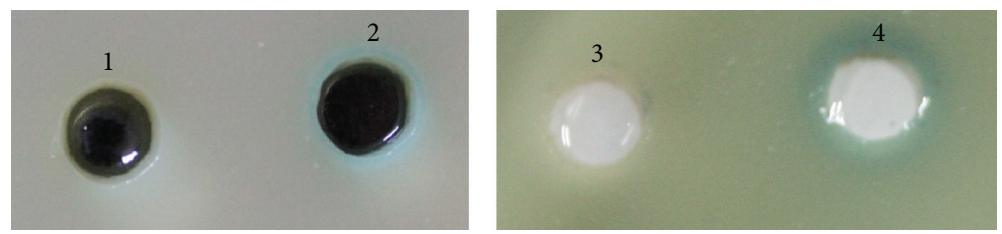

Figure 7: Measurement of the expressed B3-LAAO activity using Prussian blue agar assay [16]. Lane 1: E. coli BL21(DE3)/pET28b(+)-lao without induction; lane 2: E. coli BL21(DE3)/pET28b(+)-lao with a 6 h-induction; lane 3: E. coli BL21(DE3)/pET20b(+)-lao without induction; lane 4: E. coli BL21(DE3)/pET20b(+)-lao with a 6 h-induction.

\section{Acknowledgments}

This work was supported by the Natural Science Foundation of Zhejiang Province, China (Y5100153), the Science and Technology Planning Project of Zhejiang Province, China (Welfare Technology Applied Research Project of Zhejiang Province, Grant no. 2011C23007), and the Natural Science Foundation of ZJUT (20100213) to Zhiliang Yu.

\section{References}

[1] Z. Yu and H. Qiao, "Advances in non-snake venom l-amino acid oxidase," Applied Biochemistry and Biotechnology, vol. 167, no. 1, pp. 1-13, 2012.

[2] Y. Sakurai, H. Takatsuka, A. Yoshioka et al., "Inhibition of human platelet aggregation by L-amino acid oxidase purified from Naja naja kaouthia venom," Toxicon, vol. 39, no. 12, pp. 1827-1833, 2001.

[3] R. Iijima, J. Kisugi, and M. Yamazaki, "A novel antimicrobial peptide from the sea hare Dolabella auricularia," Developmental and Comparative Immunology, vol. 27, no. 4, pp. 305-311, 2003.

[4] M. Y. Ahn, K. S. Ryu, Y. W. Lee, and Y. S. Kim, "Cytotoxicity and L-Amino Acid Oxidase Activity of Crude Insect Drugs," Archives of Pharmacal Research, vol. 23, no. 5, pp. 477-481, 2000.

[5] O. Vallon, L. Bulte, R. Kuras, J. Olive, and F.-A. Wollman, "Extensive accumulation of an extracellular L-amino-acid oxidase during gametogenesis of Chlamydomonas reinhardtii," European Journal of Biochemistry, vol. 215, no. 2, pp. 351-360, 1993.

[6] G. M. Brearley, C. P. Price, T. Atkinson, and P. M. Hammond, "Purification and partial characterisation of a broad-range Lamino acid oxidase from Bacillus carotarum 2Pfa isolated from soil," Applied Microbiology and Biotechnology, vol. 41, no. 6, pp. 670-676, 1994.

[7] Y.-L. Huang, M. Li, Z. Yu, and P.-Y. Qian, "Correlation between pigmentation and larval settlement deterrence by Pseudoalteromonas sp. sf57," Biofouling, vol. 27, no. 3, pp. 287-293, 2011.

[8] O. Vallon, "New sequence motifs in flavoproteins: evidence for common ancestry and tools to predict structure," Proteins, vol. 38, no. 1, pp. 95-114, 2000.

[9] B. Geueke and W. Hummel, "Heterologous expression of Rhodococcus opacus L-amino acid oxidase in Streptomyces lividans," Protein Expression and Purification, vol. 28, no. 2, pp. 303-309, 2003.

[10] H. Tong, W. Chen, W. Shi, F. Qi, and X. Dong, "SO-LAAO, a novel L-amino acid oxidase that enables Streptococcus oligofermentans to outcompete Streptococcus mutans by generating $\mathrm{H}_{2} \mathrm{O}_{2}$ from peptone," Journal of Bacteriology, vol. 190, no. 13, pp. 4716-4721, 2008.
[11] H. Yang, P. M. Johnson, K.-C. Ko et al., "Cloning, characterization and expression of escapin, a broadly antimicrobial FAD-containing L-amino acid oxidase from ink of the sea hare Aplysia californica," Journal of Experimental Biology, vol. 208, no. 18, pp. 3609-3622, 2005.

[12] P. R. Kommoju, P. Macheroux, and S. Ghisla, "Molecular cloning, expression and purification of 1 -amino acid oxidase from the Malayan pit viper Calloselasma rhodostoma," Protein Expression and Purification, vol. 52, no. 1, pp. 89-95, 2007.

[13] S. Singh, B. K. Gogoi, and R. L. Bezbaruah, "Optimization of medium and cultivation conditions for L-amino acid oxidase production by Aspergillus fumigatus," Canadian Journal of Microbiology, vol. 55, no. 9, pp. 1096-1102, 2009.

[14] G. L. Smith, S. S. Socransky, and C. M. Smith, "Rapid method for the purification of DNA from subgingival microorganisms," Oral Microbiology and Immunology, vol. 4, no. 1, pp. 47-51, 1989.

[15] D. Kang, Y. S. Gho, M. Suh, and C. Kang, "Highly sensitive and fast protein detection with Coomassie brilliant blue in sodium dodecyl sulfate-polyacrylamide gel electrophoresis," Bulletin of the Korean Chemical Society, vol. 23, no. 11, pp. 1511-1512, 2002.

[16] Z. Yu, N. Zhou, C. Zhao, and J. Qiu, "In-gel determination of L-amino acid oxidase activity based on the visualization of Prussian blue-forming reaction," PLoS ONE, vol. 8, no. 2, Article ID e55548, 2013.

[17] S. G. James, C. Holmström, and S. Kjelleberg, "Purification and characterization of a novel antibacterial protein from the marine bacterium D2," Applied and Environmental Microbiology, vol. 62, no. 8, pp. 2783-2788, 1996.

[18] T. Ehara, S. Kitajima, N. Kanzawa, T. Tamiya, and T. Tsuchiya, "Antimicrobial action of achacin is mediated by L-amino acid oxidase activity," FEBS Letters, vol. 531, no. 3, pp. 509-512, 2002.

[19] T. Nishizawa, C. C. Aldrich, and D. H. Sherman, "Molecular analysis of the rebeccamycin L-amino acid oxidase from Lechevalieria aerocolonigenes ATCC 39243," Journal of Bacteriology, vol. 187, no. 6, pp. 2084-2092, 2005. 

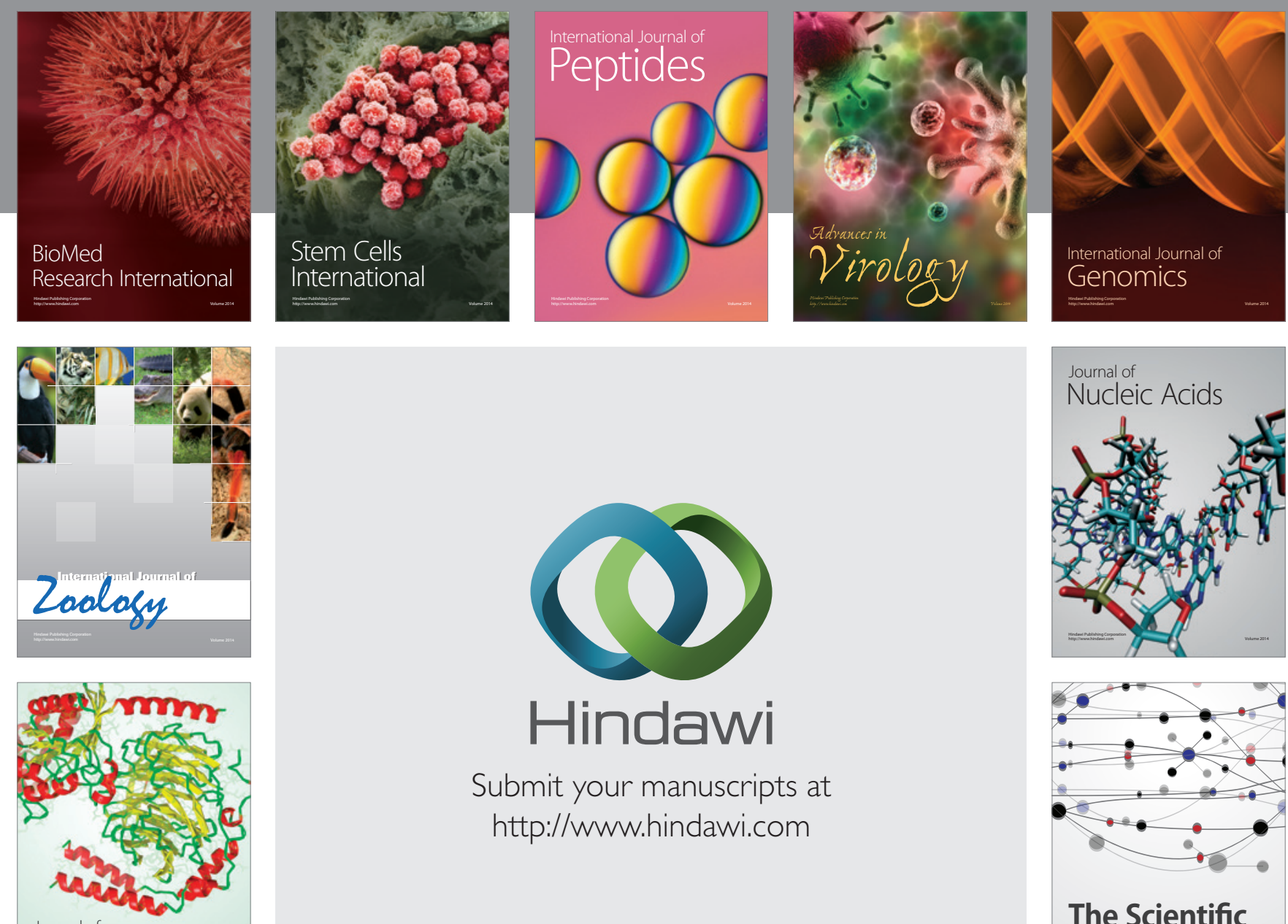

Submit your manuscripts at

http://www.hindawi.com

Journal of
Signal Transduction
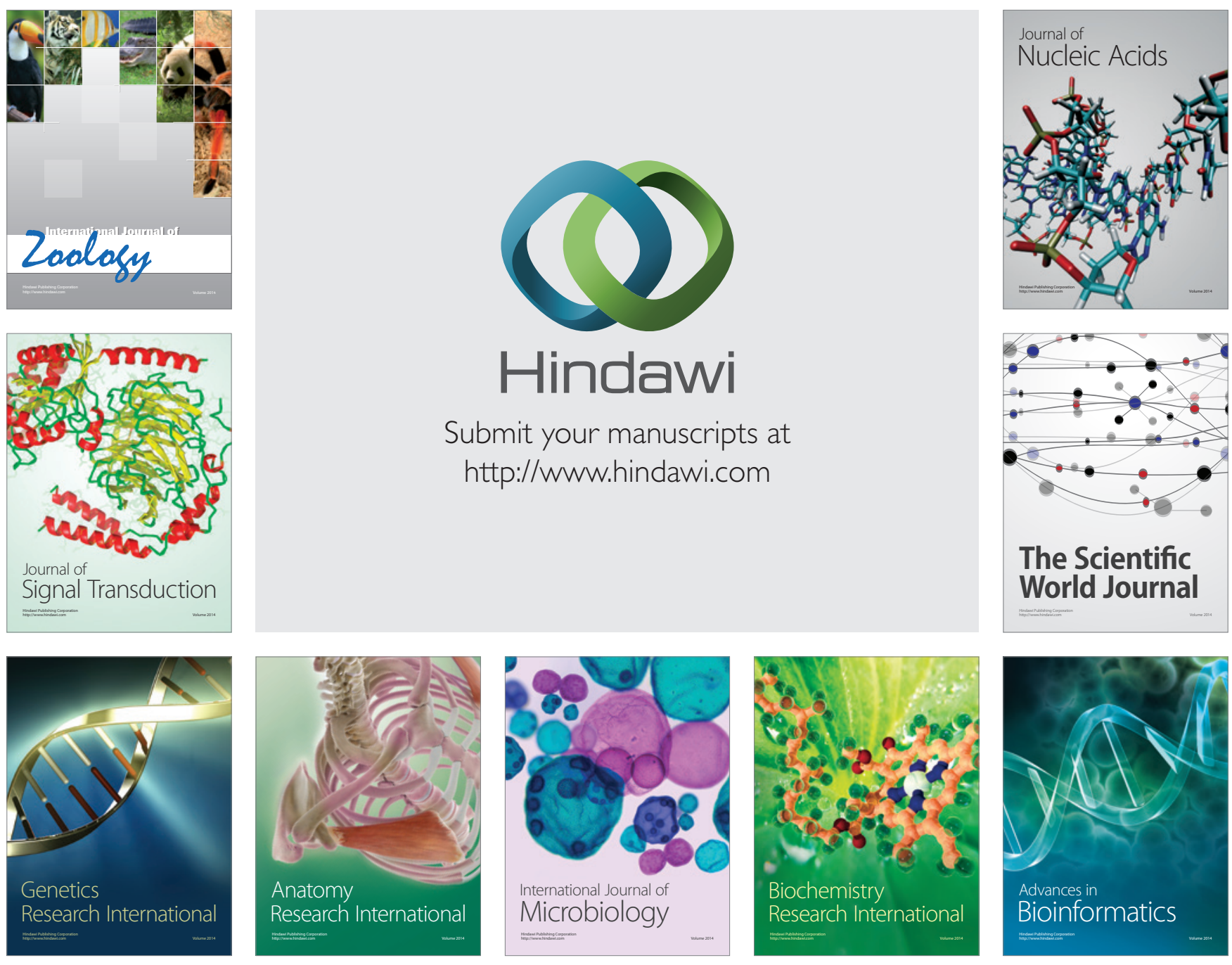

The Scientific World Journal
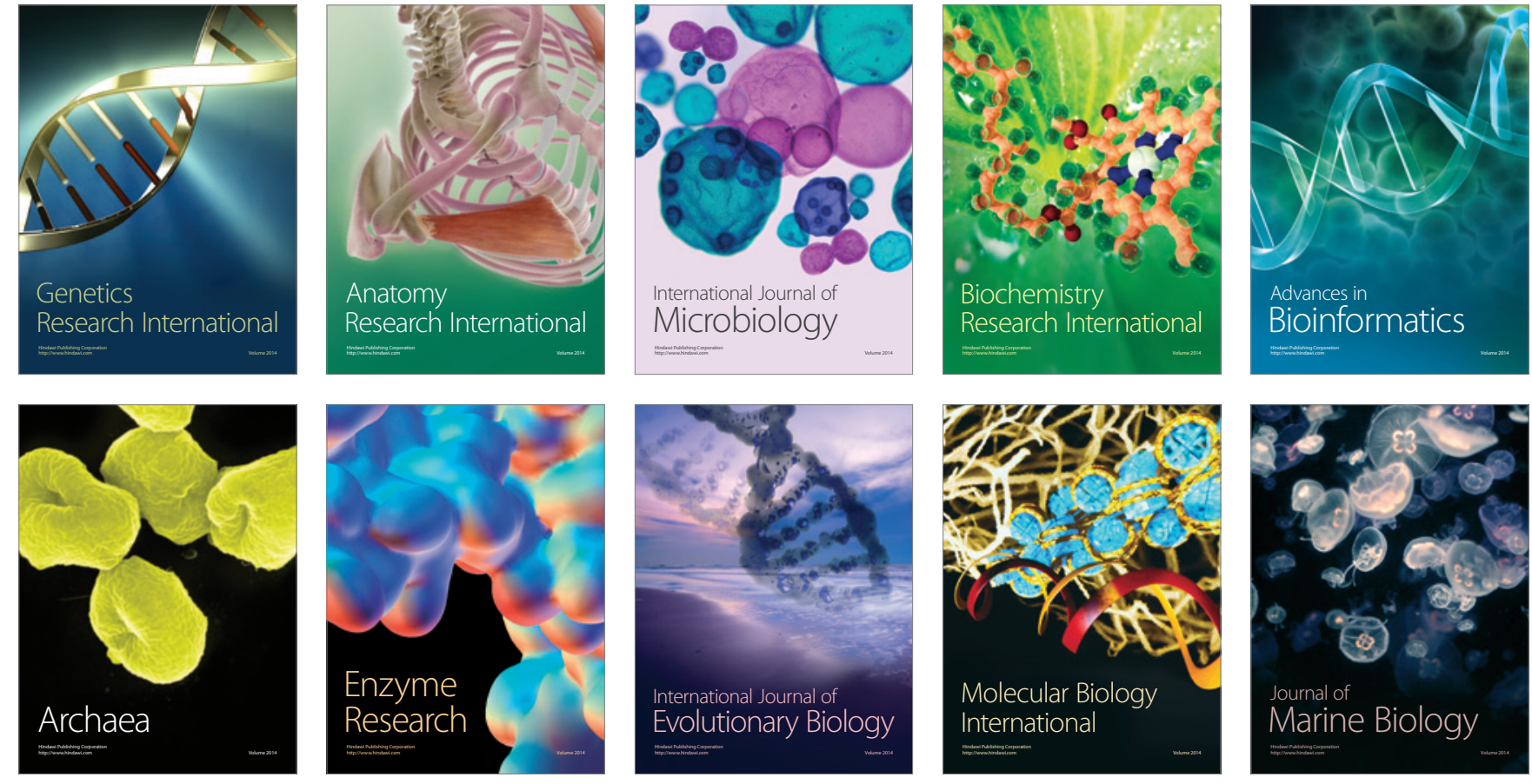